\title{
NON-LINEAR DAMPING IN THE SYSTEM OF TWO-AXIS GYROSCOPIC STABILIZER
}

\author{
J. Škoda* , J. Šklíba ${ }^{* *}$

\begin{abstract}
This paper tackles the reasons for the application of the shock absorbers in the mechanical system of the two-axis gyroscopic stabilizer. The procedure of choice of the suitable damping torque characteristics based on the numeric simulations is proposed as well.
\end{abstract}

Keywords: gyroscopic stabilizer, vibration-isolation, non-linear damper

\section{Introduction}

The effects of linear damping in the dynamical system of two-axis gyroscopic stabilizer were analyzed in Škoda et al. (2018). The cited paper concludes that the response in the supposed frequency band of excitation is worsened by additional damping. Thus, from this point of view, it is better to avoid damping. On the other hand, a certain damping is necessary to reach the asymptotic stability of equilibrium or stationary motion. A small linear damping is not sufficient to tackle impulse excitation or sudden change of the stabilizer base orientation. Thus we found the application of controlled non-linear shock absorbers as an appropriate solution.

Settings of the shock absorbers (their suitable characteristics and placing in the stabilizer) and resulting impact on the stabilizer motion will be assessed by a response on the kinematic excitation of the stabilizer base corresponding to the anticipated operating conditions. In the described case we will limit our considerations to excitation in the range of the first and second resonant frequencies of the ambulance car suspension (up to $12 \mathrm{~Hz}$ ).

\section{Preliminary considerations}

The analyzed stabilizer is intended for the controlled vibration-isolation of the sprung ambulance stretcher suspension with air springs. We have restricted analyses to three degrees of freedom of the ambulance car suspension - vertical translation and rotations about both horizontal axes. The vertical suspension is guided by the parallelogram linkage. First gimbal of the stretcher rotational suspension is pivotally connected to the upper base of the vertical guiding mechanism with the lateral rotation axis. Second gimbal is then pivoted in the first gimbal with the longitudinal rotation axis. The stretcher and both gyroscopes are attached to the second gimbal. Both gyroscopes with the vertical rotation axes are also gimballed with mutually perpendicular horizontal gimbal axes (see Fig. 1). The stabilizer is equipped by two feedbacks so called correction (torque acting on the gyroscope precession axis controlled by the displacement of respective gimbal) and compensation (torque balancing the stretcher gimbals controlled by the gyroscope precession motion). A two-axis electronic level as a sensor of stretcher orientation is attached to the second gimbal and both gyroscopes act as sensors of the disturbance torques of the stretcher suspension gimbals.

\footnotetext{
* Ing. Jan Škoda.: Technical University of Liberec, Studentská 1402/2; 461 17, Liberec 1; CZ, jan.skoda@gmail.com

** prof. RNDr. Jan Šklíba, CSc.: Technical University of Liberec, Studentská 1402/2; 461 17, Liberec 1; CZ, jan.skliba@tul.cz
} 
The controlled dampers (hydraulic or magneto-rheological) were chosen for damping of the stretcher suspension motion. It is assumed that static characteristics of the dampers is continuous and its compression and rebound parts could be different. We will try analyzing the effects of the non-linear dampers on the forced vibrations response by the numeric simulation of the stabilizer motion under the kinematic excitation of the stabilizer base. We will assume that angular momentum of both flywheels $H$ is sufficiently high (Merkin, 1974; Pelpor, 1977), which implies that the roots of characteristic equation are divided into two groups: precession which are proportional to $H^{-1}$; nutation which are proportional to $H^{1}$, their difference thus could reach several orders.

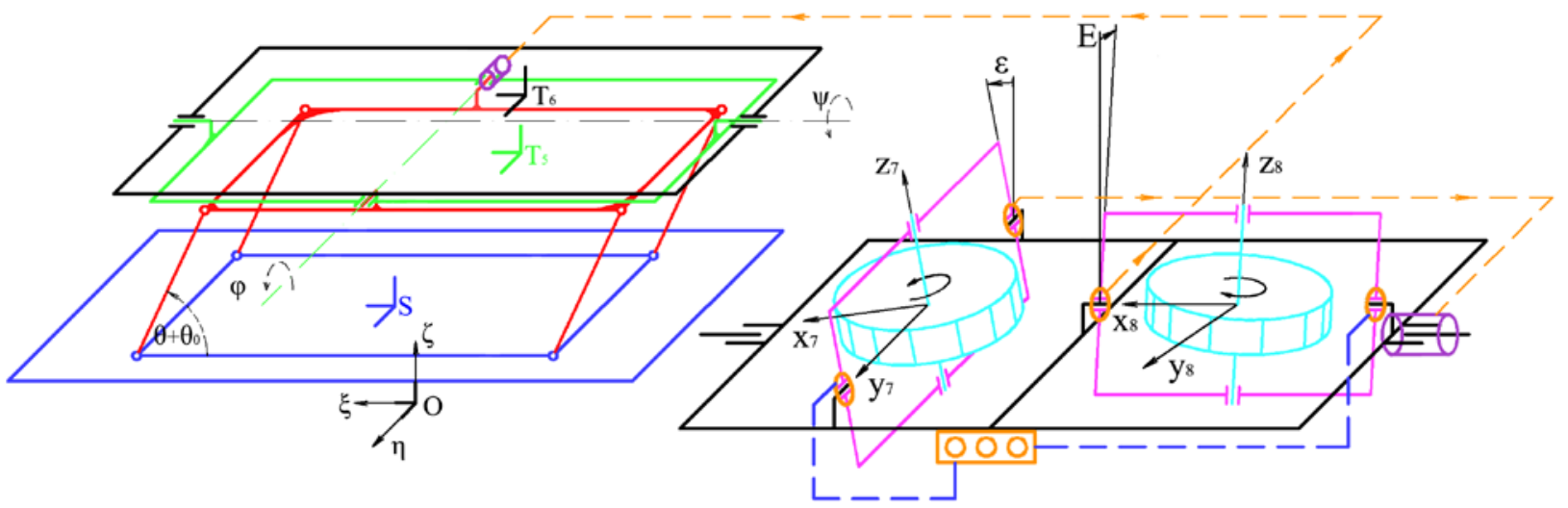

Fig. 1: Scheme of the two-axis gyroscopic stabilizer including vertical guiding mechanism.

\section{Motion equations of the system}

According to our previous work (Šklíba et al., 2018), coupling between the vertical guiding linkage and the gimbals is small, thus it is possible to analyze the motion of both subsystems separately. The system of motion equations of the two-axis gyroscopic stabilizer without the vertical guiding mechanism could be written in the following form:

$$
\mathbf{A} \ddot{\mathbf{q}}+\left(\mathbf{B}+\mathbf{B}_{1}(t)+\mathbf{G}\right) \dot{\mathbf{q}}+\left(\mathbf{C}+\mathbf{C}_{1}(t)+\mathbf{K}\right) \mathbf{q}=\mathbf{E}(t),
$$

where:

$$
\begin{aligned}
& \mathbf{A}=\left[\begin{array}{cccc}
a_{11} & a_{12} & a_{13} & 0 \\
a_{12} & a_{22} & 0 & a_{24} \\
a_{13} & 0 & a_{33} & 0 \\
0 & a_{24} & 0 & a_{44}
\end{array}\right], \mathbf{B}=\left[\begin{array}{cccc}
b_{11} & 0 & 0 & 0 \\
0 & b_{22} & 0 & 0 \\
0 & 0 & b_{33} & 0 \\
0 & 0 & 0 & b_{44}
\end{array}\right], \mathbf{G}=\left[\begin{array}{cccc}
0 & 0 & 0 & H \\
0 & 0 & H & 0 \\
0 & -H & 0 & 0 \\
-H & 0 & 0 & 0
\end{array}\right], \\
& \mathbf{C}=\left[\begin{array}{cccc}
c_{11} & 0 & 0 & 0 \\
0 & c_{22} & 0 & 0 \\
0 & 0 & c_{33} & 0 \\
0 & 0 & 0 & c_{44}
\end{array}\right], \mathbf{K}=\left[\begin{array}{cccc}
0 & 0 & 0 & k_{14} \\
0 & 0 & k_{23} & 0 \\
0 & -k_{32} & 0 & 0 \\
-k_{41} & 0 & 0 & 0
\end{array}\right],
\end{aligned}
$$

$\mathbf{B}_{1}(t)$ and $\mathbf{C}_{1}(t)$ are dependent on the rotational excitations of the stabilizer base and $\mathbf{E}(t)$ is the vector of the excitation. The vector of generalized coordinates is $\mathbf{q}=\left[\begin{array}{llll}\varphi(t) & \psi(t) & \epsilon(t) & \mathrm{E}(t)\end{array}\right]^{\mathrm{T}}$. Matrix $\mathbf{K}$ contains coefficients of so called correction $\left(k_{32}, k_{41}\right)$ and compensation $\left(k_{23}, k_{14}\right)$ feedback, $\mathbf{G}$ stands for the gyroscopic forces and $\mathbf{C}$ is the stiffness matrix. Elements of matrix $\mathbf{B}$ are linear damping slopes; $b_{11}$ and $b_{22}$ are for damping of first and second gimbal, when non-linear damping torques are applied they are replaced by functions of damping torques. The linear damping (passive resistances in bearings) of gimbals of gyroscopes is assumed to be small.

\section{Choice of damping characteristics}

The use of the shock absorber with non-symmetric characteristics is not anyhow beneficial for tackling the rotational vibrations by the gimbals (unlike for vertical vibration-isolation), therefore the magnetorheological controlled shock absorber (originally intended for the driver's seat) with symmetrical 
characteristics was chosen for further considerations, examples of its characteristics are pictured in Fig. 2. By the control of the flow of magneto-rheological fluid inside the damper cavities is affected only the slope of the static characteristics for low velocities.

It has to be said that damping in the system of gyroscopic stabilizer generally worsens the vibrationisolation performance. Furthermore high damping causes high precession displacement of the gyroscopes so it decreases the torque acting by the gyroscope on the respective stretcher suspension gimbal. On the other hand, impulse induced natural vibrations last longer when damping is low. It is obvious that a choice of suitable damping characteristics is a compromise.

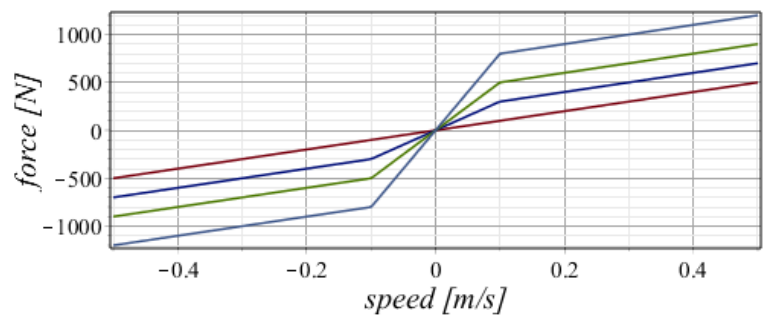

Fig. 2: Example of MR controlled damper characteristics

The benefits of the non-linear damping characteristics is depicted in Fig. 4 - time response on sudden change of the stabilizer base rotational displacement about longitudinal axis (see Fig. 3), where $\varphi$ is absolute rotational displacement of first gimbal and $\mathrm{E}$ is precession displacement with respect to its initial position. High damping (red response) worsens the vibration-isolation effect and increases the gyroscope precession movement. Decrease of damping makes the response better (see green curves), lowers the gyroscope precession motion, but transient induced oscillations last longer. Non-linear characteristics of the damper combines both aforementioned benefits - blue curves in Fig. 4 .
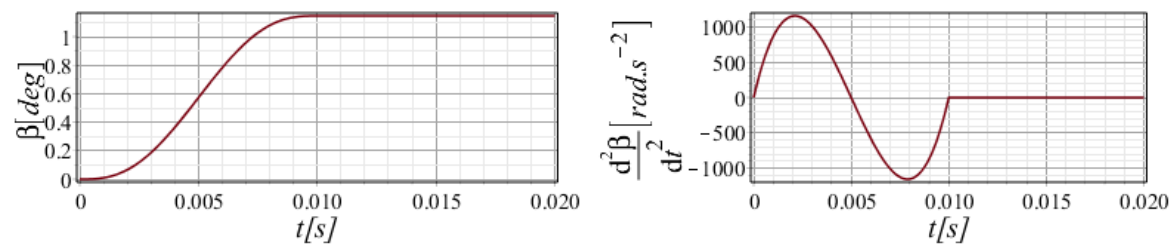

Fig. 3: Used rotational step excitation of the stabilizer base.
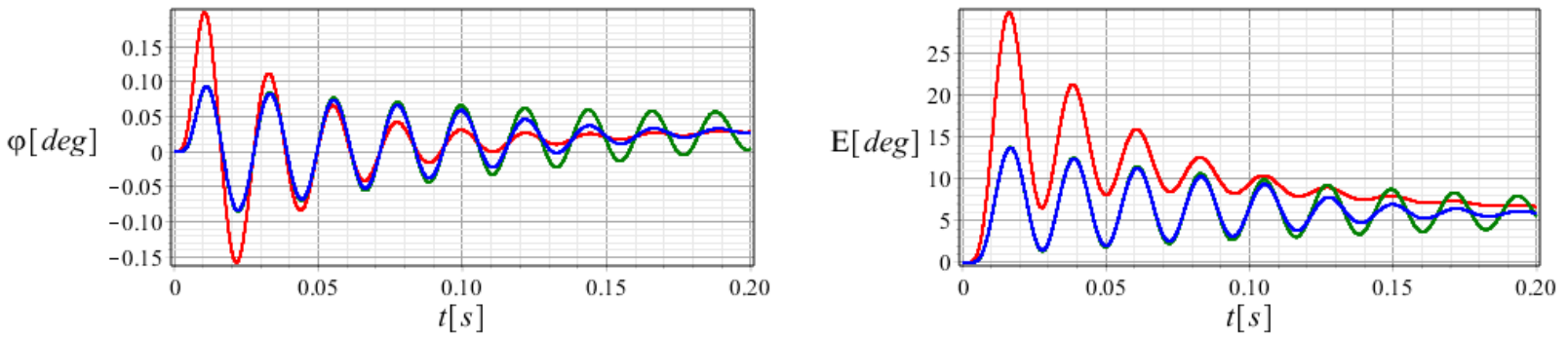

Fig. 4: Comparison of response on the step change of gyroscope base rotation (angles in deg); red linear damper; green - linear damper (lower slope); blue non-linear damper (same slope for low velocities as red linear damper).

As it was stated the abrupt change of the stabilizer base or swift impulse excitation of the highly damped gyroscopic stabilizer can cause strong induced transient oscillations. The worse disturbance could be noticed just at the moment of the abrupt change of the stabilizer base and this effect is even worse for higher damping (see Fig. 4). A solution of this issue could be the time delay of the damping torque lowering damping effect of the shock absorber e.g. when a control system detects a steep gradient of the stabilizer base rotational movement. The demonstration is pictured in the Fig. 5: red - low damping; green - high damping; blue - delayed high damping; excitation by $20 \mathrm{~ms}$ lasting impulse rotational movement of the stabilizer base with maximal displacement approx. 5.7 deg. By this example could be suggested the application of the controlled damper and development of its control. 

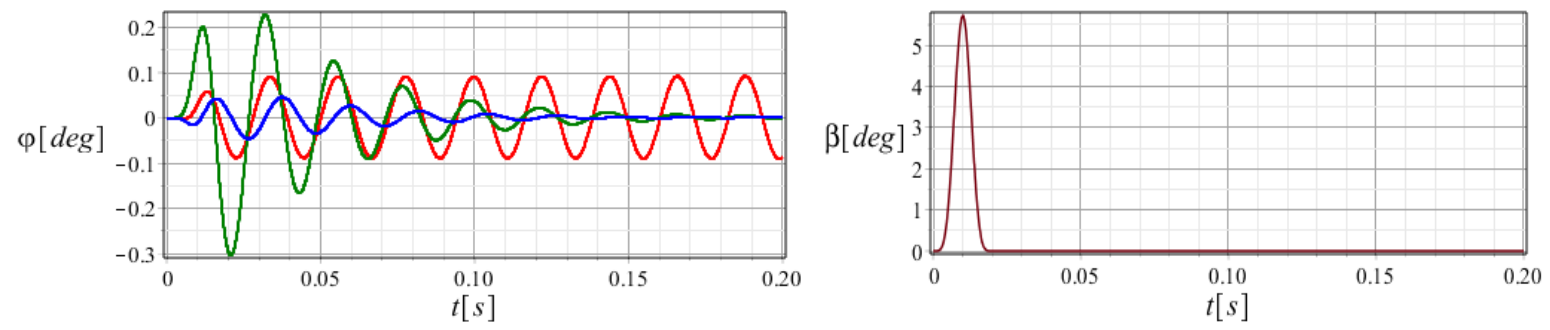

Fig. 5: Demonstration of delayed damping positive effect - impulse response of first gimbal; excitation signal (right).

Finally it has to be said that the suggested process of the suitable damper choice depends on e.g. purpose and other properties of the stabilizer. Due to high order of the system and a number of system parameters it is very troublesome to optimize the system analytically.

\section{Conclusions}

Based on the analyses of the gyroscopic stabilizer dynamic system and its numeric simulations we can conclude the following:

1. By the analysis of the numerical simulations of the response to the excitation (impulse, step) it is possible to find out suitable damping torque characteristics.

2. Slight damping (approximately $10^{-4}$ of the stretcher gimbals damping) in bearings of the gyroscope precession axis also positively affects a decay of transient induced vibrations of the system.

3. Mitigation of unfavorable effect of damping on the response of the stabilized gimbal to the abrupt excitation is possible by controlling of the shock absorber characteristics or by the time delay of damping torque.

4. Optimization of the stabilizer behavior in a number of situations is always a trade-off decision.

\section{Acknowledgement}

This article was written at the Technical University of Liberec, Faculty of Mechanical Engineering with the support of the Institutional Endowment for the Long Term Conceptual Development of Research Institutes, as provided by the Ministry of Education, Youth and Sports of the Czech Republic in the year 2018.

\section{References}

Merkin, D. R. (1974) Gyroscopic systems. Nauka, Moscow (in Russian).

Pelpor, D. S. (1977) Design of gyroscopic systems I and II, Vysshya shkola, Moscow (in Russian).

Šklíba, J., Sivčák, M., Škoda, J. (2011) Comparison of attitudes to solve a vibration-isolation system with a gyroscopic stabilization. in: Proc. 13th World Congress in Mechanism and Machine Science, IFToMM, Guanajuato, Mexico.

Škoda, J., Šklíba, J. (2018) Viscous Friction in the Axes of Gyroscopic Stabilizer. Colloquium Dynamics of Machines and Mechanical Systems with Interactions, Institute of Thermomechanics of the CAS, Prague. 